\title{
Monohydroxybutylmercapturic Acid
}

National Cancer Institute

\section{Source}

National Cancer Institute. Monohydroxybutylmercapturic Acid. NCI Thesaurus. Code C107143.

A modified form of acetylcysteine that is a metabolite of 1,3-butadiene and may be found in the urine of smokers. 